\title{
Effet des insecticides, des variétés de chou et des dates de semis sur Plutella xylostella (L.) (Lepidoptera : Plutellidae) dans les hautes terres de l'Ouest Cameroun
}

\author{
Edwige Sidoine DJOMAHA * et T. Richard GHOGOMU \\ University of Dschang, Faculty of Agronomy and Agricultural Science, Department of Plant Protection, \\ Laboratory of Entomology, B.P: 222 Dschang, Cameroon. \\ *Auteur correspondant ; E-mail: djomahaedwige@gmail.com; sidoine.djomaha@univ-dschang.org; \\ Tél: (+237) 699789866/674505391.
}

\section{REMERCIEMENTS}

Nous sommes reconnaissants au couple TCHATCHOUA N. pour le financement de ce travail.

\section{RESUME}

Le but de ce travail a été d'étudier l'effet des insecticides, des variétés de chou et des dates de semis sur la teigne du chou à Dschang. Bacillus thuringiensis a été pulvérisé sur le chou pour contrôler les insectes. Deux insecticides conventionnels (Cyperdim 220 EC et Cypercal 100 EC) ont été utilisés comme produits comparatifs. Les variétés chou, Green Coronet et Marché de Copenhague ont été testées. Le dispositif expérimental a été en blocs aléatoires complets avec quatre répétitions. Le comptage direct des insectes a été fait hebdomadairement jusqu'à deux semaines avant la récolte. L'abondance des insectes, les dégâts, les rendements et les pertes ont été évalués. Les dégâts moyens et les pertes moyennes en 2008 ont été moins importants avec Biobit que Cyperdim et Cypercal. Le nombre de teigne en 2008 (247,5) a été plus important qu'en 2009 (2,6). Green Coronet a hébergé plus de teigne (182,6) que Marché de Copenhague (67,5). Mais son rendement moyen $(96,58 \mathrm{t} / \mathrm{ha})$ a été significativement plus élevé que celui de Marché de Copenhague $(67,15$ t/ha). Biobit a été efficace comme insecticide. Green Coronet est tolérante. Son usage peut être combiné avec l'application de Biobit en période de forte infestation de la teigne à Dschang.

(C) 2016 International Formulae Group. All rights reserved.

Mots clés: Chou, Biobit, teigne du chou, lutte intégrée, Cypercal 100EC, Cyperdim 220EC.

\section{The effect of insecticides, cabbage varieties and planting date on Plutella xylostella (L.) (Lepidoptera : Plutellidae) in the Western Highland of Cameroon}

\begin{abstract}
The study aimed at identifying the effects of insecticides, cabbage varieties and planting date on diamondback moth (DBM) in Dschang. Biobit (Bacillus thuringiensis) was applied on cabbage leaves to control insects. Two conventional insecticides Cyperdim 220 EC and Cypercal 100 EC were used as
\end{abstract}


comparative products. The cabbage varieties Green Coronet and Copenhague Market were compared. The randomised complete blocks design with 4 replications was used. Insects were directly counted, once a week till two weeks before the harvest time. DBM abundance, damage and percentage losses were obtained. The mean damage and percentage losses in 2008 were significantly low with Biobit than Cypercal and Cyperdim. The mean number of DBM in 2008 (247.5) was higher than in 2009 (2.6). Green Coronet carried more DBM (182.6) than Copenhague Market (67.5). Nevertheless, the average yield of Green Coronet was higher (96.5 t/ha) than Copenhague Market (67.15 t/ha). . This study demonstrated that Biobit, an organic insecticide do better than synthetic ones, cabbage planted during the raining season is less attacked than dry season planting and that the variety Green Coronet produce better. These three methods can be combined effectively to control DBM infestation in Dschang.

(C) 2016 International Formulae Group. All rights reserved.

Keywords: Cabbage, Biobit, diamondback moth, IPM, Cypercal 100EC, Cyperdim 220EC.

\section{INTRODUCTION}

Les fruits et les légumes sont d'importantes composantes des systèmes agricoles. Ils constituent des sources de fibres, de vitamines et de sels minéraux dans l'alimentation et contribuent ainsi à la lutte contre la malnutrition (Kam, 2005). Le chou comme toutes les crucifères est caractérisé par sa richesse en fibre végétale, vitamines, minéraux et oligoéléments (Pamplona-Roger, 2002). La production camerounaise de chou est essentiellement celle des hautes terres de l'Ouest comprenant les régions de l'Ouest et du Nord-Ouest où le climat assez froid favorise sa culture. Cette production a été de $37000 \mathrm{t}$ sur 3000 ha avec un rendement de 12,3 t/ha en 2007 (FAO, 2007).

La culture du chou comme d'autres spéculations connaît des contraintes majeures de production telles que les maladies et les ravageurs (Charleston et al., 2005). Les insectes constituent le groupe de ravageurs dont les dégâts sont importants à cause des conditions climatiques favorables à leur développement (Imam et al., 2010 ; Walangululu et Mushagalusa, 2000). Parmi les cinq principaux ravageurs de chou, Plutella xylostella (L.) est le grand dévastateur et sévit dans sept pays de l'Afrique de l'Est et du Sud (Tewary et al. 2005 ; Walangululu et Mushagalusa, 2000). La teigne du chou (Lepidoptera : Plutellidae) est un ravageur de crucifères dans le monde (Dixon, 2007;
Pichon et al., 2006). Les pertes dues à $P$. xylostella sont très importantes; $52 \%$ ont été rapportées en Inde (Krishnamoorthy, 2004).

La lutte la plus préconisée est l'usage des insecticides et les matières actives utilisées sont : la cyperméthrine, l'endosulfan, le diméthoate, la deltaméthrine, le malathion et l'alphaméthrine (Hasheela et al., 2010; Martin et al., 2006 ). Le coût de contrôle de la teigne du chou dans le monde est important. L'argent employé pour l'achat des insecticides augmente le coût de production et diminue les marges de bénéfices. Parmi les inconvénients liés à l'utilisation des insecticides s'ajoute le phénomène de résistance à différentes matières actives jusqu'ici testées dans toutes les parties du monde (Bautista et al., 2007 ; Joia et al., 2004). Ainsi, la lutte contre la teigne du chou est rendue difficile avec les insecticides conventionnels. Les cas de résistances ont été signalés au Japon, en Hawaï, en Australie et en Chine (Oddhiambo et al., 2010).

Cette résistance à plusieurs pesticides a donné lieu à la recherche des méthodes alternatives, principalement la lutte intégrée. Selon Dixon (2000), certaines variétés de chou présentent des propriétés antixénose et antibiotique limitant les dégâts de la teigne du chou. Des études faites par Munthali (2009) ont révélé par exemple que les cultivars de chou ayant des feuilles épaisses et une forte concentration en eau et en chlorophylle 
hébergent plus de pucerons. Hasheela et al. (2010) au Kenya affirment que les variétés de chou Pruktor F1 et Marché de Copenhague hébergent moins de larves de la teigne du chou tant dans les parcelles traitées au Diméthoate que les parcelles non traitées. Pour Ali et al. (2000), le chou Colza est très résistant aux pucerons. Leslie et al. (2009) ont trouvé que les pluies entraînent une forte mortalité des larves et des pupes de la teigne du chou pendant la grande saison de pluies plus que pendant la petite saison de pluies à cause de l'effet mécanique des gouttelettes d'eau sur les feuilles. En République Démocratique du Congo, la saison de novembre à février est plus propice au développement des ravageurs du chou (Walangululu et Mushagalusa, 2000). Toutefois, en zone tropicale et subtropicale, la lutte chimique demeure le moyen de contrôle le plus usuel. Mais leur usage se fait en combinaison avec d'autres méthodes de lutte (Martin et al., 2006 ).

De nos jours, les nouveaux composés utilisés dans la lutte chimique en général et la lutte chimique contre les ravageurs du chou en particulier sont ceux qui affectent le processus de développement des insectes tels que l'inhibition de la synthèse de la chitine, le mimétisme de l'hormone juvénile, les inhibiteurs de l'ecdysone, de la respiration, du récepteur nicotine acétylcholine, de la sécrétion des glandes salivaires chez les insectes (Martin et al., 2006 ). Hasheela et al. (2010) ont décelé que l'application du Diméthoate réduit significativement les larves de la teigne du chou. Les études de Martin et al. (2006) soulignent que $P$. xylostella ne s'observent pas dans les parcelles de chou recouvertes de moustiquaires imprégnées de deltamethrine ( $12 \mathrm{~g} \mathrm{~m} . \mathrm{a} / \mathrm{l})$.

$\mathrm{Ce}$ travail a pour objectifs de déterminer l'efficacité des deux insecticides conventionnels et un biopesticide (Bacillus thuringiennsis) ainsi qu'évaluer les dates de semis et la susceptibilité de deux variétés sur la population de la teigne du chou à Dschang.

\section{MATERIEL ET METHODES}

Cette étude a été conduite en deux dates de semis de septembre 2008 à décembre 2008 et de mars 2009 à juin 2009 à Dschang $\left(5^{\circ} 24^{\prime} 23^{\prime \prime}-5^{\circ} 26^{\prime} \quad 23^{\prime \prime} \quad \mathrm{N}\right.$, latitude et $10^{\circ} 04^{\prime} 05^{\prime},-10^{\circ} 07^{\prime} 43^{\prime}$ 'E, longitude, $1440 \mathrm{~m}$ d'altitude). La température moyenne de la zone d'étude est de $19,87^{\circ} \mathrm{C}$ et l'humidité relative de l'air est au dessus de $60 \%$. Les précipitations moyennes annuelles sont comprises entre 1800 et $2000 \mathrm{~mm}$.

Deux variétés de chou, Green Coronet (hybride tropicale (4 mois) de haute altitude à pomme compacte de forme semi-globe) et Marché de Copenhague (précoce (3 mois) à feuilles sessiles et vert brillant) ont été utilisées. Ce sont des variétés les plus connues et cultivées par les paysans dans la zone d'étude.

Les essais ont débuté par la mise en place d'une pépinière. L'objectif était d'obtenir les plants vigoureux pour la transplantation et assurer les remplacements avec les plants de même âge. La planche de pépinière a mesuré $5 \mathrm{~m}^{2}$. Le sol a été amendé à base de fientes de poule à la dose de $5 \mathrm{t} / \mathrm{ha}$ et traité au VYDATE 240 EC (Oxamyl $240 \mathrm{~g} / \mathrm{l}$ ) à la dose de $10 \mathrm{~g}$ de produit commercial par $\mathrm{m}^{2}$ contre les nématodes et insectes telluriques. Le semis a été effectué 7 jours après l'amendement sur des lignes séparées de $15 \mathrm{~cm}$ et à $1 \mathrm{~cm}$ de profondeur. La pépinière a été arrosée tous les matins exceptés les jours de pluie. Une semaine après le semis, les plants ont reçu un traitement fongicide de prévention au manèb appliqué à la dose de 1 $\mathrm{kg} / \mathrm{ha}$ contre les maladies fongiques. Un désherbage s'est effectué deux semaines après le semis. Cette dernière opération a été couplée à l'apport d'un engrais foliaire (Fertigofol) de NPK, 20-20-20 appliqué à la dose de $1 \mathrm{~kg} / \mathrm{ha}$. La transplantation a été faite manuellement, 27 jours après le semis en 
pépinière au stade 4 à 5 vraies feuilles. Seuls les plants vigoureux et sains ont été prélevés. Les unités expérimentales (parcelles) ont reçu un amendement à base de fientes de poule à la dose de $5 \mathrm{t} / \mathrm{ha}$. L'application a été faite en bande sur les lignes de semis. Cette phase s'est déroulée une semaine avant la transplantation pour permettre la décomposition des fientes. Un engrais composé de NPK (18-11-12) a été répandu à la dose $300 \mathrm{~kg} / \mathrm{ha}$ deux semaines après la transplantation. Le deuxième apport a eu lieu quatre semaines après le premier et est constitué de l'engrais foliaire 20-20-20 (NPK) appliqué à la dose de $1 \mathrm{~kg} / \mathrm{ha}$. Le sarclage manuel a eu lieu une semaine après la transplantation et les deux autres toutes les trois semaines.

Le dispositif expérimental a été en blocs aléatoires complets avec quatre répétitions. La surface totale mise en valeur est de $437 \mathrm{~m}^{2}$. Chaque bloc a eu 4 parcelles de $20 \mathrm{~m}^{2}$ avec 1,25 m d'espacement entre elles pour gérer les pertes d'insecticides. Les écartements entre les plants ont été de 50 X 50 $\mathrm{cm}$, soit une densité de 40000 plants/ha. Chaque parcelle a porté deux variétés. Les insecticides ont été appliqués à l'aide d'un pulvérisateur à dos $\left(\begin{array}{ll}15 & 1\end{array}\right)$, une fois par semaine dès la deuxième semaine après transplantation. L'opération a eu lieu tôt le matin en temps non venteux. Les doses d'application des insecticides ont été de 0,36 1/ha pour le Cypercal 100 EC, 1,5 1/ha pour le Cyperdim $220 \mathrm{EC}$ et $1 \mathrm{~kg} / \mathrm{Ha}$ pour le Biobit (Bacillus thuringiensis variété kurstaki). Le traitement fongicide a été effectué une fois par semaine avec le mancozeb (Dose de 2,5 $\mathrm{kg} / \mathrm{ha}$ ) ou du chlorothalonyl $500 \mathrm{~g} / \mathrm{l}$ et $100 \mathrm{~g} / \mathrm{l}$ de carbendazim (Banko+: dose de 1 1/ha). Trois sarclages manuels ont été réalisés. L'irrigation s'est faite manuellement trois fois par semaine avec un arrosoir.

La collecte des données a commencé deux semaines après la transplantation. Les données ont été collectées sur huit (8) plantes par variété, situées à l'intérieur de la parcelle. Le comptage des chenilles et des chrysalides de la teigne du chou a été fait une fois par semaine et un jour avant le traitement insecticide jusqu'à deux semaines avant la récolte. Il a été question d'examiner les faces inférieures et supérieures des feuilles ainsi que les nervures principales des plantes choisies. Pour quantifier les dégâts, une échelle de dégâts décrite par Nagawa (2003) a été utilisée. Il s'agit :

$1=$ pas de dégâts $2=<25 \%$ de la surface foliaire détruite

$3=25-50 \%$ de la surface foliaire détruite

$4=51-75 \%$ de la surface foliaire détruite

$5=>75 \%$ de la surface foliaire détruite

A la maturité, les têtes de chou ont été collectées, pesées et le poids moyen a été calculé. Le rendement a été déterminé pour chaque variété en fonction des traitements par la formule suivante :

Le poids moyens des têtes à la récolte a été obtenu par la division de la somme de poids de quatre têtes de chou sur quatre.

Le pourcentage de perte à la récolte (\%) a été calculé par la soustraction du poids moyens à la récolte au poids moyen commercialisable $(\mathrm{kg})$ des têtes de chou dépourvues de feuilles endommagées par les chenilles, les pucerons et les autres ravageurs sur le poids moyens à la récolte.

\section{Analyses statistiques}

Les données ont été saisies sur Excel. Celles relatives à l'évaluation de la population de $P$. xylostella et aux rendements des variétés ont fait l'objet d'une analyse factorielle de la variance. Les moyennes ont été séparées par le test de la Plus Petite Différence Significative (PPDS) au seuil $5 \%$ de probabilité. Le logiciel d'analyse statistique MSTATC (Michigan State University) a été utilisé. 


\section{RESULTATS}

\section{Abondance des chenilles et des chrysalides de Plutella xylostella}

Les chenilles et les chrysalides ont été présentes sur les plants de chou dès le début des collectes. Le nombre moyen de $P$. xylostella par plante en septembre-décembre 2008 était de 247,5 significativement $(P \leq$ $0,05)$ plus important qu'en mars-juin 2009 $(2,6)$. Quant aux variétés, Green Coronet a hébergé en moyenne 182,6 teignes de chou par plant contre 67,5 chez Marché de Copenhague. Pendant la période de forte infestation (2008), l'application des insecticides a réduit significativement les effectifs de la teigne par variété dans les parcelles traitées au Biobit $(26,0 ; 8,6)$ que les parcelles non traitées $(212,3 ; 50,8)$ (Tableau 1). Par ailleurs, le nombre moyen d'insectes dans les parcelles de Biobit et témoins a été moins élevé que celui de Cyperdim $220 \mathrm{EC}$ $(668,0 ; 262,0)$ et de Cypercal 100 EC $(540,0$; $211,1)$ comparables entre elles. En 2009, les différences significativement $(P \leq 0,05)$ n'ont pas existé entre les traitements quelles que soit les variétés (Tableau 1). Biobit a été ainsi plus efficace que Cyperdim 220 EC et de Cypercal $100 \mathrm{EC}$.

\section{Les dégâts moyens de la teigne}

La présence des chenilles et chrysalides de la teigne du chou sur les plantes de chou s'est matérialisées par la perforation des feuilles. Le pourcentage de surface foliaire totale endommagée a varié de 0 à $50 \%$ (Figure 1, Tableau 2). Ces dégâts ont été observés sur les deux variétés de chou. En 2008, le traitement Biobit a présenté moins de dégâts moyens $(2,0 ; 2,5)$ que le témoin $(3,0)$. Les dégâts moyens dans les parcelles non traitées et les parcelles traitées aux Cypercal 100 EC et Cyperdim $220 \mathrm{EC}$ ont été semblables $(3,0)$ (Tableau 2). En 2009, les dégâts moyens ont été moins importants et similaires dans tous les traitements. Biobit a été meilleur que Cypercal 100 EC et Cyperdim 220 EC.
Rendement moyen des variétés par date de semis et par traitement insecticide

Les rendements moyens ont varié de 84 à 107 t/ha pour la variété Green Coronet contre 48 à 76 t/ha pour la variété Marché de Copenhague (Tableau 3). En 2008, le rendement moyen a été moins important (74,90 t/ha) qu'en 2009 (88,83 t/ha). Green Coronet a eu un rendement moyen $(96,58$ t/ha) significativement plus élevé que Marché de Copenhague $(67,15$ t/ha). En 2008, les parcelles de Green Coronet traitées au Biobit ont présenté des rendements moyens (94,62 t/ha) importants que le témoin ( $88,10 \mathrm{t} / \mathrm{ha})$. Le rendement moyen du témoin est comparable à ceux de Cypercal et de Cyperdim (84,78 t/ha ; 91,17 t/ha) respectivement. En 2009, les parcelles de Green Coronet non traitées ont eu un rendement moyen plus élevé (107,32 t/ha) que ceux des parcelles traitées aux insecticides Cypercal, Cyperdim et Biobit respectivement $(104,25 \mathrm{t} / \mathrm{ha} ; 99,22 \mathrm{t} / \mathrm{ha}$; 103,22 t/ha). La variété Green Coronet a hébergé plus de teigne de chou en 2008 et son rendement moyen en 2008 et 2009 est meilleur que celui de Marché de Copenhague. Elle se présente ainsi comme une variété tolérante.

\section{Les pertes moyennes en rendement}

Les pertes moyennes en rendement des variétés Green Coronet et Marché de Copenhague ont varié de 29 à $48 \%$ au cours des saisons dans les parcelles traitées et non traitées (Tableau 4). En 2009, il n'y a pas eu différences significatives entre les traitements insecticides et le témoin. Par contre en 2008, le traitement Biobit a enregistré moins de perte moyenne en rendement sur les deux variétés de chou que le témoin et les deux autres traitements (Cypercal et Cyperdim). Marché de Copenhague a eu la plus grande perte moyenne dans les parcelles non traitées que les parcelles traitées. Marché de Copenhague est donc une variété susceptible en période de forte infestation. 
Tableau 1: Nombre moyen de Plutella xylostella par plant par traitements insecticides au cours des saisons 2008 et 2009 en fonction des variétés de chou.

\begin{tabular}{lcccc}
\hline Traitement & \multicolumn{2}{c}{ septembre- décembre 2008 } & \multicolumn{2}{c}{ mars- juin 2009 } \\
\cline { 2 - 5 } insecticide & Green & Marché de & Green & Marché de \\
& Coronet & Copenhague & Coronet & Copenhague \\
Témoin & $212,3_{\mathrm{c}}$ & $50,8_{\mathrm{b}}$ & $5,1_{\mathrm{a}}$ & $4,3_{\mathrm{a}}$ \\
Cypercal & $540,0_{\mathrm{b}}$ & $211,1_{\mathrm{a}}$ & $1,5_{\mathrm{a}}$ & $1,0_{\mathrm{a}}$ \\
Cyperdim & $668,0_{\mathrm{a}}$ & $262,0_{\mathrm{a}}$ & $1,7_{\mathrm{a}}$ & $1,0_{\mathrm{a}}$ \\
Biobit & $26,0_{\mathrm{d}}$ & $8,6_{\mathrm{b}}$ & $5,1_{\mathrm{a}}$ & $1,5_{\mathrm{a}}$ \\
\hline Les moyennes suivies par la même lettre dans la même colonne ne sont pas significativement différentes selon le test de \\
la PPDS (P=0.05). CV: $52,72 \%$.
\end{tabular}

Tableau 2: Dégât moyen de Plutella xylostella par plante par traitements insecticides au cours des saisons de culture en fonction des variétés de chou.

\begin{tabular}{lcccc}
\hline $\begin{array}{l}\text { Traitement } \\
\text { insecticide }\end{array}$ & \multicolumn{2}{c}{ septembre à décembre 2008 } & \multicolumn{2}{c}{ mars à juin 2009 } \\
\cline { 2 - 5 } & Green Coronet & $\begin{array}{c}\text { Marché de } \\
\text { Copenhague }\end{array}$ & Green Coronet & $\begin{array}{c}\text { Marché de } \\
\text { Copenhague }\end{array}$ \\
\hline Témoin & $3,0_{\mathrm{a}}$ & $3,0_{\mathrm{a}}$ & $2,5_{\mathrm{a}}$ & $2,2_{\mathrm{a}}$ \\
Cypercal & $3,0_{\mathrm{a}}$ & $3,0_{\mathrm{a}}$ & $2,0_{\mathrm{a}}$ & $2,0_{\mathrm{a}}$ \\
Cyperdim & $3,0_{\mathrm{a}}$ & $3,0_{\mathrm{a}}$ & $2,0_{\mathrm{a}}$ & $2,0_{\mathrm{a}}$ \\
Biobit & $2,5_{\mathrm{b}}$ & $2,0_{\mathrm{ab}}$ & $2,2_{\mathrm{ab}}$ & $2,0_{\mathrm{a}}$ \\
\hline Les moyennes suivies par la même lettre dans la même colonne ne sont pas significativement différentes selon le test de la \\
PPDS (P=0.05) CV:10,80\%.
\end{tabular}

Tableau 3: Rendement moyen (t/ha) des variétés de chou au cours des saisons 2008 et 2009 en fonction des traitements insecticides.

\begin{tabular}{lcccc}
\hline Traitement & \multicolumn{2}{c}{ septembre à décembre 2008 } & \multicolumn{2}{c}{ mars à juin 2009 } \\
\cline { 2 - 5 } insecticide & $\begin{array}{c}\text { Green } \\
\text { Coronet }\end{array}$ & $\begin{array}{c}\text { Marché de } \\
\text { Copenhague }\end{array}$ & Green Coronet & $\begin{array}{c}\text { Marché de } \\
\text { Copenhague }\end{array}$ \\
Témoin & $88,10_{\mathrm{b}}$ & $48,17_{\mathrm{c}}$ & $107,32_{\mathrm{a}}$ & $73,77_{\mathrm{a}}$ \\
Cypercal & $84,78_{\mathrm{b}}$ & $68,82_{\mathrm{a}}$ & $104,25_{\mathrm{ab}}$ & $74,47_{\mathrm{a}}$ \\
Cyperdim & $91,17_{\mathrm{ab}}$ & $63,47_{\mathrm{ab}}$ & $99,22_{\mathrm{b}}$ & $71,47_{\mathrm{a}}$ \\
Biobit & $94,62_{\mathrm{a}}$ & $60,15_{\mathrm{b}}$ & $103,22_{\mathrm{ab}}$ & $76,90_{\mathrm{a}}$ \\
\hline \multicolumn{2}{l}{ Les moyennes suivies par la même lettre dans la même colonne ne sont pas significativement différentes selon le test de la } \\
PPDS (P=0.05). CV:7,63\%.
\end{tabular}

Tableau 4: Pourcentage de perte moyenne en rendement des variétés de chou par traitements insecticides au cours des saisons 2008 et 2009.

\begin{tabular}{lcccc}
\hline Traitement & \multicolumn{2}{c}{ septembre à décembre 2008 } & \multicolumn{2}{c}{ mars à juin 2009 } \\
\cline { 2 - 5 } insecticide & Green Coronet & $\begin{array}{c}\text { Marché de } \\
\text { Copenhague }\end{array}$ & Green Coronet & $\begin{array}{c}\text { Marché de } \\
\text { Copenhague }\end{array}$ \\
\hline Témoin & $36,79_{\mathrm{ab}}$ & $48,19_{\mathrm{a}}$ & $31,25_{\mathrm{a}}$ & $30,23_{\mathrm{a}}$ \\
Cypercal & $38,09_{\mathrm{ab}}$ & $41,62_{\mathrm{b}}$ & $30,23_{\mathrm{a}}$ & $32,24_{\mathrm{a}}$ \\
Cyperdim & $38,65_{\mathrm{a}}$ & $32,52_{\mathrm{d}}$ & $31,28_{\mathrm{a}}$ & $30,55_{\mathrm{a}}$ \\
Biobit & $34,50_{\mathrm{b}}$ & $36,48_{\mathrm{c}}$ & $29,26_{\mathrm{a}}$ & $31,76_{\mathrm{a}}$ \\
\hline
\end{tabular}

Les moyennes suivies par la même lettre dans la même colonne ne sont pas significativement différentes selon le test de la PPDS $(\mathrm{P}=0.05) \mathrm{CV}: 10,93 \%$. 


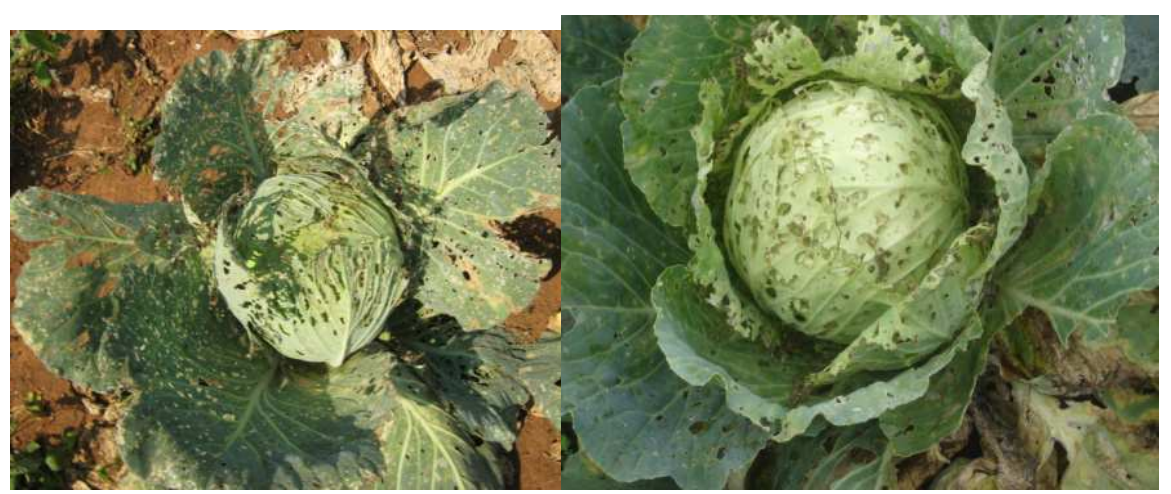

Figure 1: Dégâts de la teigne en champ sur les variétés de chou Green Coronet (à gauche) et Marché de Copenhague (à droite) à maturité.

\section{DISCUSSION}

Plusieurs auteurs ont travaillé sur l'effet des insecticides, des variétés de chou et des dates de semis sur la population de la teigne du chou (Hasheela et al., 2010; Munthali, 2009 ; Dixon, 2007 ; Dixon, 2000 ; Walangululu et Mushagalusa, 2000). L'incidence des ravageurs est généralement liée au stade de développement de la plante hôte. Jouer sur la date de semis ou de transplantation permet de séparer la période de sensibilité de la plante hôte et la période d'abondance des ravageurs (Stoll, 2002). Leslie et al. (2009) ont montré que les pluies entraînent une forte mortalité des larves et pupes de la teigne du chou en grande saison de pluies plus qu'en petite saison de pluies à cause de l'effet mécanique des gouttelettes d'eau sur les feuilles. Walangululu et Mushagalusa (2000) ont rapporté que les pics de populations des ravageurs de chou se situaient en saison sèche. Les résultats de la présente étude ont révélé que la saison de septembre à décembre 2008 a été plus propice au développement de la teigne et cette période est transitoire entre la fin des pluies et la misaison sèche dans la zone d'étude. La période de mars à juin a eu un effet positif sur la population de Plutella xylostella.
Certaines variétés de chou présentent des propriétés antixénose et antibiotique limitant les dégâts des pucerons et de la teigne du chou (Dixon, 2007). Selon la FAO (2000), il existe des variétés résistantes, tolérantes et sensibles aux ravageurs du chou. Une variété résistante ne permet pas le développement normal des insectes. Ceci peut être dû aux poils absorbants longs ou épais qui empêchent le déplacement des insectes ou limite leur nutrition ou bien dû à la sécrétion des toxines ou de la composition chimique de la sève qui rend ces plantes moins attractives aux ravageurs. Une variété tolérante est infestée par les insectes mais les symptômes sont moins sévères. Le rendement n'est pas affecté ou est très peu affecté. Une variété sensible quant à elle est très affectée au niveau du rendement et même de la qualité marchande des têtes de chou. Dans cette étude, Green Coronet a été plus attaquée que Marché de Copenhague mais son rendement moyen était élevé montrant ainsi sa tolérance aux attaques de la teigne du chou. Wanko (2008) a trouvé que le rendement de Green Coronet était plus élevé (96,4 t/ha) que celui de Marché de Copenhague (56,6 t/ha) à Dschang.

Les résultats de ce travail ont révélé que Biobit plus meilleur que Cypercal 100 EC 
et Cyperdim 220 EC. Le nombre moyen de teigne par plante, les dégâts moyens, les rendements moyens et les pertes moyennes au cours des deux périodes de semis ont été meilleurs avec Biobit que ceux obtenus avec Cypercal 100 EC et Cyperdim 220 EC. Ces résultats corroborent avec de Capinera (2008) qui a rapporté que Biobit (Bacillus thuringiensis) détruit les insectes. Odhiambo et al., 2010 ont trouvé que la teigne du chou était résistante à tous les insecticides surtout les pyréthrinoïdes. En Chine, Sengonga et Lui (2002) décèlent que la population de la teigne $\mathrm{du}$ chou et du puceron du chou atteignait 39,7 et 68,3 individus/30plantes dans le traitement par GCSC-BtA respectivement. Ces données sont 1,5 et 11 fois moins importantes que celles obtenues dans le traitement au Méthomyl. Le fait que Biobit ne soit pas efficace au même titre que Cypercal 100 EC et Cyperdim 220 EC en 2009 peut être dû à l'effet négatif des pluies. Ceci a été rapporté par Dixon (2000) qui a découvert que les pluies compromettent l'efficacité de Bacillus thuringiensis car elles entraînent les fortes mortalités de chenilles et chrysalides.

\section{Conclusion}

Les résultats de ce travail indiquent que Biobit, insecticide biologique à base du Bacillus thuringiensis est efficace contre la teigne du chou. Ils montrent aussi que la culture du chou peut se faire sans traitement insecticide en mars-juin et que la variété Green Coronet est tolérante aux attaques de la teigne du chou et est plus productive quelle que soit la date de semis. De ce qui précède, on peut conseiller aux paysans de choisir la variété Green Coronet et d'associer le traitement insecticide Biobit dans la pratique de cette culture.

\section{CONFLICT D'INTERETS}

Les auteurs ne déclarent aucun conflit d'intérêts.

\section{CONTRIBUTIONS DES AUTEURS}

ESD a conduit le travail et rédigé le manuscrit ; TRG a supervisé le travail.

\section{REMERCIEMENTS}

Nous remercions les techniciens de laboratoire d'entomologie de la Faculté d'Agronomie et des Sciences Agricoles (FASA) pour leur soutien dans la mise en place du dispositif expérimental. Un grand merci à Mlle NGATAT Sergine pour les conseils qui ont amélioré la qualité de ce travail. Pendant la rédaction de cet article, nous avons bénéficié de l'encouragement et des suggestions de plusieurs collaborateurs. Merci à Dr. MEUTCHIEYE F. et Mr. ZANGUE M.B.

\section{REFERENCES}

Bautista MAM, Tanaka T, Miyata T. 2007. Identification of permethrininducible cytochrome P450s from the diamondback moth, Plutella xylostella (L.) and the possibility of involvement in permethrin resistance. Pest. Biochem. Physiol., 87: 85-93. DOI : 10.1016/j.ibmb.2008.09.005.

Capinera JL. 2008. Encyclopedia of entomology $\left(2^{\text {nd }}\right.$ edn, Vol 4) (S-Z). Springer; 4411p.

Charleston DS, Kfir R, Dicke MV, Le M. 2005. Impact of botanical pesticides derived from Melia azadarache and Azadirachta indica on the biology of two parasitoid species of the diamondback moth. Biological Control, 33: $131-142$.

Dixon AFG. 2000. Insect Predator-Prey Dynamics: Ladybird Beetles and Biological Control. Cambridge University Press: Cambridge, UK; $255 \mathrm{p}$.

Dixon AFG. 2007. Insect Predator-Prey Dynamics: ladybird Beetles and 
Biological Control. Cambridge University Press: New York; 268 p.

FAO. 2000. Cabbage integrated pest management: an ecological guide, FAO, 206p.

FAO. 2007. www.fao.org/waicent, Septembre 2008.

Hasheela EBS, Nderitu JH, Olubayo FM, Kasina M. 2010. Evaluation of cabbage varietal resistance to diamondback moth (Plutella xylostella) infestation and damage. Tunisian Journal of Plant Protection, 5: 91-98. DOI: http://www.iresa.agrinet.tn/tjpp/tjpp9/tj pp9/9Eddie1.pdf.

Imam TS, Yusuf AU, Mukhtar MD. 2010. A survey of some insect pests of cultivated vegetables in three selected irrigation areas along Jakara river, Kano, Nigeria. Int. J. Biol. Chem. Sci., 4(2): 400-406, DOI: http://ajol.info/index.php/ijbcs.

Joia K, Suri S, Udeaan AS. 2004. Baseline Susceptibility of Diamondback Moth, Plutella xylostella (Linn.), to New Insecticides. Department of Entomology Punjab Agricultural University Luthiana: 1410004 India.

Kam DR. 2005. Inventaire des parasitoïdes de Plutella xylostella sur la culture du chou Brassica oleracea L. dans les localités de Babadjou et Santa, Mémoire d'Ingénieur Agronome, Université de Dschang, Cameroun, 120 p.

Krishnamoorthy A. 2004. Biological control of diamondback moth Plutella xylostella (L.), an Indian scenario with reference to past and future strategies. pp. 204-211 In Proceedings of the International Symposium, 21-24 October 2002 (A.A.Kirk, D.Bordat Eds.), Montpellier, France, CIRAD.

Martin T, Assogba-Komlan F, Houndete T, Hougard JM \& Chandre F. 2006. Insect
Proof Net on cabbage in Africa: a tool for small-scale producers to increase sustainability of growing vegetables practices. Journal of Economic Entomology, 99(2): 450-454. DOI : http://www.mivegec.ird.fr/images/stori es/PDF_files/1203.pdf

Moussavou G. 2007. Identification des ennemis naturels de Plutella xylostella ravageur du chou (Brassica sp.) dans la localité de Dschang, Cameroun, Mémoire d'Ingénieur Agronome, Université de Dschang, Cameroun. $116 \mathrm{p}$.

Munthali DC. 2009. Evaluation of cabbage varieties for resistance to cabbage aphid. African Entomology, 17(1): 1-7. DOI: http://dx.doi.org/10.4001/ 003.017.0101.

Nagawa F. 2003. Incidence of diamondback mot Plutella xylostella and its parasitoids on cabbage in Uganda, MSc thesis, Makéréré University, Uganda. $78 \mathrm{p}$.

Odhiambo JAO, Gbewonyo WSK, Obengofori D, Wilson MD, Boakye DA and Brown C. 2010. Resistance of diamondback moth to insecticides in selected cabbage farms in southern Ghana. Int. J. Biol. Chem. Sci., 4(5): 1397-1409, DOI: http://ajol.info/index.php/ijbcs.

Pamplona-Roger GD. 2002. Santé par les Aliments. $4^{\mathrm{e}}$ Impression de la $1^{\text {ere }}$ Edition en Langue Française. Madrid ; $381 p$.

Pichon A, Arvanitakis L, Roux O, Kirk AA, Alauzet C, Bordat D, Legal L. 2006. Genetic differentiation among various populations of the diamondback moth, Plutella xylostella (Lepidotera: Yponomeutidae). Bulletin Entomologcal Research, 96: 137-144.

Sengonga C \& Liu B. 2002. Effect of GCSCBtA biocide on population dynamics of 
cabbage pests and their natural enemies from field in Southeastern China. $J$. Pest. Science, 75: 46-50.

Stoll G. 2002. Protection Naturelle des Végétaux en Zones Tropicales. Vers une Dynamique de l'Information. CTA: Margraf Verlag, Allemagne ; 386p.

Tewary DK, Bhardwaj A and Shanker A. 2005. Pesticide activities in five medicinal plants collected from mid hill of Western Himalayas. Industrial Crops and Products. Journal of Applied Bioscience, 3(4): 411-418. DOI: 10.1016/j.indcrop.2005.01.004
Walangululu JM, Mushagalusa GN. 2000. Les principaux ravageurs des choux pommés (Brassica oleracea var. capitata subs sabouda) à Bukavu et ses environs. Tropicultura, 18(2): 55-57. DOI :www.tropicultura.org/text/v18n2/ 55.pdf.

Wanko TR. 2008. Influence de différents types de bio fertilisants sur la performance du chou (Brassica oleracea) dans les hauts plateaux de l'Ouest Cameroun. Mémoire d'Ingénieur Agronome. Université de Dschang, Cameroun, 80p. 